\title{
A intervenção fonoaudiológica no pós-operatório da hipertrofia benigna do músculo masseter
}

\author{
Laura Davison Mangilli*, Christiane Siman Rodrigues*, Alcione Ramos Campiotto**
}

\begin{abstract}
Resumo
Objetivo: descrever a abordagem fonoaudiológica no pós-operatório de pacientes que realizaram correção cirúrgica da hipertrofia do músculo masseter. Metodologia: foram coletados dados referentes à avaliação e ao tratamento fonoaudiológico de 4 prontuários de sujeitos de ambos os gêneros, com faixa etária entre 16 e 24 anos, com hipertrofia benigna do músculo masseter, tratados cirurgicamente. Resultados: as principais queixas pós-cirúrgicas estiveram relacionadas à limitação da abertura da boca, à dor na região da cirurgia, à rigidez muscular e a estalo em região da ATM. A terapia fonoaudiológica baseou-se em orientação quanto à retirada de hábitos deletérios; termoterapia na região do músculo masseter; manipulação da musculatura envolvida extra e intrabucais; alongamento da musculatura facial e cervical; alavanca de abertura forçada de boca e exercícios de órgãos fonoarticulatórios. Conclusões: a terapia fonoaudiológica apresenta-se como uma possibilidade de complementação ao tratamento cirúrgico, na adequação da amplitude dos movimentos mandibulares, assim como na eliminação dos sintomas presentes no pós-cirúrgico e na conscientização dos hábitos deletérios, que são apontados pela literatura como possíveis fatores desencadeantes.
\end{abstract}

Palavras-chave: Hipertrofia benigna de músculo masseter. Reabilitação miofuncional oral. Tratamento fonoaudiológico.

\section{INTRODUÇÃO}

A hipertrofia do músculo masseter (HMM) é uma condição benigna caracterizada por um desenvolvimento excessivo, uni ou bilateral, do músculo masseter, cuja incidência é maior na $2^{\mathrm{a}} \mathrm{e}$ $3^{\text {a }}$ décadas de vida, ocorrendo com a mesma freqüência nos dois gêneros ${ }^{1,4,5,6,7,8,9,10,12,13,15,16,17,19,20}$.

Associado a esta patologia é possível verificarse um aumento secundário no ângulo da mandíbula (esporão ósseo), ocasionado pela remodela- ção funcional das forças de inserção do músculo

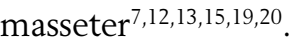

A HMM pode ser erroneamente diagnosticada como parotidites, neoplasias malignas ou tumores benignos, raramente é acompanhada de dor e, na maioria das vezes, os pacientes procuram tratamento por razões estéticas 9,10,11,12,16,18.

O tratamento da hipertrofia do músculo masseter consiste de técnicas cirúrgicas e não cirúrgicas, denominadas também de conservadoras $1,3,5,17,20$.

* Fonoaudiólogas, alunas do Curso de Aperfeiçoamento em Motricidade Oral em Fonoaudiologia da Irmandade da

Santa Casa de Misericórdia de São Paulo.

** Fonoaudióloga, Coordenadora do Curso de Aperfeiçoamento em Motricidade Oral em Fonoaudiologia da Irmandade

da Santa Casa de Misericórdia de São Paulo. 
Muitos autores citam em seus trabalhos a possibilidade da utilização de ambos os tratamen$\operatorname{tos}^{1,3,9,11,20}$. No entanto, o tratamento cirúrgico é preconizado pela maioria dos autores ${ }^{2,4,8,9,13,17,18} \mathrm{e}$ consiste na ressecção do feixe profundo do músculo masseter e do esporão ósseo ${ }^{1,3,8,9,13,17}$, podendo ser realizado por acesso extrabucal ${ }^{1,20}$ ou intrabucal ${ }^{13}$.

Garcez e Santos ${ }^{1}$ apontaram a vantagem estética da técnica cirúrgica intrabucal, porém afirmaram que com o uso da incisão extrabucal há um melhor acesso e visualização de todo o ângulo mandibular, mantendo-se, assim, a cadeia de assepsia mais segura. Destacaram a necessidade de futuras pesquisas com a técnica intrabucal para se atingir a sua comprovação.

Considerando, então, a necessidade de uma reabilitação miofuncional oral pós-operatória, o objetivo deste estudo foi descrever a abordagem fonoaudiológica realizada em pacientes que apresentavam hipertrofia benigna do músculo masseter, tratados cirurgicamente.

\section{REVISÃO DA LITERATURA}

A etiologia desta patologia ainda é desconhecida $^{3,4,5,15,16,17,18}$ e muitos autores acreditam que esteja associada a uma atividade aumentada do músculo masseter, por apertamento dentário, bruxismo, má oclusão, problemas periodontais, disfunção temporomandibular, mastigação unilateral, mastigação de chicletes e mastigação de alimentos mais duros $3,4,5,6,7,8,9,10,11,12,15,16,17,18,19,20$

Mandel, Kaynar'; Hakam, Beheiry, Helmy3; Mandel e Tharakan ${ }^{8}$ relataram a possibilidade de uma etiologia congênita para a hipertrofia do músculo masseter. Trujillo, Fontão e Souza ${ }^{20}$ acreditaram que usualmente a hipertrofia poderia estar associada a uma predisposição genética.

Outros autores acreditam que fatores emocionais ${ }^{1,7,11}$, reações psicológicas ${ }^{20}$ e distúrbios psicológicos ${ }^{10}$ podem também estar associados a esta patologia.

Garcez, Santos ${ }^{1}$; Trujillo, Fontão e Souza ${ }^{20}$ sugeriram que para se estabelecer o diagnóstico da hipertrofia do músculo masseter é necessária uma boa anamnese e histórico clínico, complementados por um exame clínico criterioso e por exames de imagem para confirmação dos achados clínicos. Sano et al. ${ }^{14}$ relataram que normalmente as imagens dessa lesão são as radiografias e a tomografia computadorizada. Morse e Brown ${ }^{10}$ afirmaram que as radiografias e sialografias podem ajudar no diagnóstico, porém o único exame específico é a tomografia computadorizada.

Segundo Newton, Cowpe e McLure ${ }^{12}$, o diagnóstico da hipertrofia do músculo masseter deve ser feito clinicamente e usualmente confirmado com alguma intervenção cirúrgica. Investigações incluindo radiografias, sialografias, tomografias computadorizadas e ultra-sonografias podem auxiliar o diagnóstico.

Sano et al. ${ }^{14}$ defenderam também o uso da ressonância magnética, da tomografia computadorizada e da ultra-sonografia no período pré-operatório, com intuito de ajudar na determinação da ressecção, diminuir o tempo da cirurgia, minimizar a perda de sangue e produzir melhores resultados. Newton, Cowpe e McLure ${ }^{12}$ também observaram que a tomografia computadorizada fornece uma excelente visão da anatomia do músculo e possibilita uma melhor mensuração da área a ser seccionada.

Já para Mandel e Tharakan ${ }^{8}$, a tomografia computadorizada e a ressonância magnética são exames que podem oferecer informações quando o diagnóstico clínico não foi possível.

Greenwood $^{2}$ incluiu as fotografias antigas e recentes como mais uma forma de auxiliar o diagnóstico.

Trujillo, Fontão e Souza ${ }^{20}$ afirmaram que, após o diagnóstico, o paciente deve ser encorajado a diminuir a excitabilidade e a tensão muscular, e que a primeira escolha deve ser a terapia conservadora.

De acordo com To et al. ${ }^{19}$; Trujillo, Fontão e Souza $^{20}$ o tratamento cirúrgico apresenta como risco a anestesia geral, as hemorragias pós-operatórias, o edema, as infecções, a cicatriz e as lesões no nervo facial. 
Outra forma de tratamento é a não cirúrgica ou conservadora. $\mathrm{O}$ ajuste oclusal, a terapia de placas miorrelaxantes, a terapia de relaxamento, a terapia medicamentosa (espasmódicos, tranqüilizantes e antidepressivos) e a utilização de injeções da toxina botulínica tipo A são exemplos deste tipo de tratamento ${ }^{5,8,9,11,17,19,20}$.

A utilização de injeções da toxina botulínica do tipo A no músculo masseter é citada por alguns autores como uma alternativa de tratamento $^{5,8,9,11,17,19}$.

Moore e Wood ${ }^{11}$ sugeriram que esta técnica leva à atrofia muscular e torna o músculo inapto. $\mathrm{O}$ retorno da atividade muscular ocorre quando uma nova terminação nervosa se forma, restabelecendo a transmissão neuro-muscular.

Para $\operatorname{Smyth}^{17}$, o uso de injeções locais de pequenas doses da toxina botulínica no músculo ocasiona paralisia local do mesmo, pois há uma desenervação funcional com subseqüente atrofia muscular.

Já Mandel e Tharakan ${ }^{8}$ acreditavam que os hábitos deletérios seriam eliminados pela dificuldade de ativação muscular proporcionada pela ação da toxina botulínica e, assim, a forma original do músculo poderia ser retomada. Citaram como pontos negativos a possibilidade de tolerância do corpo à toxina, se utilizada por um longo tempo e o preço alto do tratamento.

To et al. ${ }^{19}$ concluíram que o tratamento com a toxina botulínica é uma boa alternativa, mas que o efeito é temporário e que injeções são necessárias para manter a atrofia do músculo.

Mandel e Kaynar ${ }^{7}$ relataram que o exame histológico do tecido removido durante a cirurgia aponta apenas o aumento das fibras musculares em tamanho e diâmetro, com resultado de biópsia normal.

Satoh et al. ${ }^{15}$ descreveram a atividade muscular, o metabolismo e a composição do tipo das fibras do músculo masseter usando a eletromiografia (EMG), a P-magnetic spectroscopy (MRS) e a enzyme-histochemistry.

A EMG não apontou hiperatividade e a P-MRS mostrou espectro de energia normal. A composi- ção do tipo das fibras apresentou grande diferença em relação ao músculo sadio, demonstrando perda das fibras do tipo II B, um aumento das fibras do tipo II A e do tipo I M e II C e uma diminuição das fibras do tipo I.

Segundo Hakam, Beheiry, Helmy ${ }^{3}$ e Lasco ${ }^{4}$, as alterações decorrentes do tratamento cirúrgico são o edema e a limitação da abertura oral. Para o segundo autor, esses sintomas tendem a diminuir no espaço de três a quatro semanas, quando se restabelecem a plenitude dos movimentos mandibulares e a capacidade mastigatória. Já para os primeiros autores a introdução de exercícios passivos e ativos para abertura de boca é de extrema importância já no quarto dia de pós-operatório, para que estes sintomas sejam eliminados o mais breve possivel.

Skoura et al. ${ }^{16}$ discutiram um caso de hipertrofia do músculo masseter associada ao uso de anabolizantes e à realização da mastigação unilateral por um período de um mês. Apontam que esta patologia nunca fora discutida com ocorrência dentro de um curto período de tempo e, desta forma, defendem que o uso de anabolizantes, associado à mastigação unilateral pode ter sido a causa da hipertrofia do músculo masseter, uma vez que esta substância aumenta o metabolismo muscular.

\section{MATERIAL E MÉTODO}

Para a realização desta pesquisa foram coletados os dados de quatro prontuários de sujeitos de ambos os gêneros, com faixa etária entre 16 e 24 anos, com diagnóstico de hipertrofia benigna do músculo masseter adquirida (Tab. 1).

Tabela 1 - Distribuição dos sujeitos segundo faixa etária e gênero.

\begin{tabular}{cccc}
\hline Caso & Paciente & Gênero & Idade \\
$\mathbf{1}$ & D.P.B.J. & $\mathrm{M}$ & 19 \\
$\mathbf{2}$ & J. L. & $\mathrm{F}$ & 16 \\
$\mathbf{3}$ & A.F.P.B. & $\mathrm{M}$ & 24 \\
$\mathbf{4}$ & J.C. & $\mathrm{M}$ & 24 \\
\hline
\end{tabular}


Estes pacientes haviam sido encaminhados para avaliação e conduta fonoaudiológica no Setor de Terapia Fonoaudiológica da Irmandade da Santa Casa de Misericórdia de São Paulo, pelo Setor de Cirurgia Buco Maxilo Facial deste mesmo hospital. É importante salientar que todos os pacientes já haviam sido tratados cirurgicamente e apresentavam como queixa principal dor e limitação da abertura de boca.

Foram retirados dos prontuários dados referentes à avaliação e ao tratamento fonoaudiológico realizados em cada um dos pacientes.

A avaliação fonoaudiológica se restringiu aos aspectos miofuncionais orais e constou de duas etapas: a anamnese e o exame clínico.

Durante a anamnese foram coletados os dados de identificação, queixa, história pregressa da queixa - hábitos deletérios (bruxismo, trismo, hábito de mascar chicletes), mastigação unilateral, fatores emocionais, antecedentes familiares, hábitos alimentares anteriores à cirurgia e atuais, presença de parestesia e/ou dor, e dados sobre má oclusão e/ou disfunção da articulação temporomandibular fornecidos pelo odontólogo.

O exame clínico foi iniciado com a avaliação dos aspectos morfológicos dos órgãos fonoarticulatórios (lábios, língua, bochechas, palato, mandíbula, dentes, oclusão e disfunção da ATM), seguida da avaliação do sistema sensório motor oral (mobilidade, tonicidade, sensibilidade e dor à palpação) e funções neurovegetativas (respiração, mastigação, deglutição e fala, sendo que a mastigação e a deglutição foram avaliadas de forma subjetiva, ou seja, conforme a descrição do paciente).

Os materiais utilizados para o exame clínico foram luva, espátulas, paquímetro, lanterna e espelho de Glatzel.

Após a avaliação fonoaudiológica os pacientes iniciaram terapia fonoaudiológica semanal individual, na qual se enfatizou: orientação quanto à retirada de hábitos deletérios; compressa quente e úmida na região do músculo masseter; manipulação da musculatura envolvida extra e intrabucais; alongamento da musculatura facial e cervical; alavanca de abertura forçada de boca manual e com espátulas; exercícios de órgãos fonoarticulatórios - movimentos mandibulares dirigidos, exercícios de língua associados à movimentação da mandíbula para correção do desvio, quando necessário.

\section{RESULTADOS}

Serão apresentados os dados de caracterização da amostra referentes à distribuição dos achados de acordo com o procedimento cirúrgico (tempo de pós-operatório e tipo de procedimento cirúrgico), queixa, hábitos deletérios, medidas de abertura de boca inicial e final com respectivo número de sessões de terapia fonoaudiológica. Esses dados foram distribuídos conforme demonstra a tabela 2 .

O procedimento cirúrgico realizado em 3 casos (Caso 2, 3 e 4) foi o de remoção do feixe profundo dos músculos masseteres bilateralmente, sendo que no caso 3 também foi realizada ostectomia oblíqua direita. No caso 1, o procedimento cirúrgico realizado foi o de remoção do feixe profundo do músculo masseter unilateral esquerdo mais ostectomia do mesmo lado.

As principais queixas relatadas pelos pacientes estiveram relacionadas à limitação da abertura da boca, à dor na região da cirurgia, à rigidez muscular e a estalo na região da ATM, independente da cirurgia realizada.

Os hábitos deletérios referidos pelos pacientes, e possiveis fatores desencadeantes, foram o apertamento dentário e o bruxismo.

A abertura de boca inicial, mensurada durante a avaliação, variou entre 15 e $24 \mathrm{~mm}$, sendo que em dois pacientes observou-se desvio sistemático para um dos lados.

O número mínimo de terapias fonoaudiológicas foi de 6 sessões e o máximo de 10 sessões, num tempo mínimo de dois meses e máximo de três meses.

Esse tempo de atendimento foi determinado pelo ganho de abertura de boca considerado satis- 
Tabela 2 - Caracterização da amostra.

\begin{tabular}{|c|c|c|c|c|c|c|c|c|}
\hline Caso & Paciente & $\begin{array}{l}\text { Encami- } \\
\text { nhamento }\end{array}$ & $\begin{array}{l}\text { Técnica cirúrgica } \\
\text { realizada }\end{array}$ & Queixa & $\begin{array}{l}\text { Hábitos dele- } \\
\text { térios }\end{array}$ & $\begin{array}{l}\text { Medida abertura } \\
\text { inicial }\end{array}$ & $\begin{array}{l}\text { Medida abertura } \\
\text { final }\end{array}$ & $\begin{array}{l}\text { Terapia fonoau- } \\
\text { diológica }\end{array}$ \\
\hline 1 & D.P.B.J. & Buco & $\begin{array}{l}\text { P0 de } 27 \text { dias } \\
\text { de remoção do } \\
\text { feixe profundo } \\
\text { do m. masseter } \\
\text { esquerdo + } \\
\text { ostectomia }\end{array}$ & $\begin{array}{l}\text { Limitação } \\
\text { da abertura } \\
\text { de boca }\end{array}$ & Apertar dentes & $\begin{array}{l}20 \mathrm{~mm} \text { c/ desvio } \\
\text { para a esquerda }\end{array}$ & $40 \mathrm{~mm} \mathrm{~s} /$ desvio & 9 sessões \\
\hline 2 & J.L. & Buco & $\begin{array}{l}\text { P0 de } 15 \text { dias de } \\
\text { remoção do feixe } \\
\text { profundo do m. } \\
\text { masseter bilateral }\end{array}$ & $\begin{array}{l}\text { Dor na } \\
\text { região da } \\
\text { operação } \\
\text { e rigidez } \\
\text { muscular }\end{array}$ & $\begin{array}{c}\text { Bruxismo e } \\
\text { apertar dentes }\end{array}$ & $18 \mathrm{~mm}$ & $37 \mathrm{~mm}$ & 10 sessões \\
\hline 3 & A.F.P.B. & Buco & $\begin{array}{l}\text { P0 de } 15 \text { dias de } \\
\text { remoção do feixe } \\
\text { profundo do m. } \\
\text { masseter bilateral } \\
\text { + ostectomia } \\
\text { direita }\end{array}$ & $\begin{array}{l}\text { Dor e ruído } \\
\text { articular }\end{array}$ & $\begin{array}{c}\text { Bruxismo e } \\
\text { apertar dentes }\end{array}$ & $24 \mathrm{~mm}$ & $40 \mathrm{~mm}$ & 6 sessões \\
\hline 4 & J.C. & Buco & $\begin{array}{l}\text { PO de } 2 \text { meses de } \\
\text { remoção do feixe } \\
\text { profundo do m. } \\
\text { masseter bilateral, } \\
\text { pior à esquerda }\end{array}$ & $\begin{array}{l}\text { Limitação da } \\
\text { abertura de } \\
\text { boca e dor } \\
\text { no músculo } \\
\text { masseter }\end{array}$ & Apertar dentes & $\begin{array}{c}15 \mathrm{~mm} \text { com desvio } \\
\text { para esquerda }\end{array}$ & $39 \mathrm{~mm}$ & 9 sessões \\
\hline
\end{tabular}

fatório, que variou de 37 a 40mm, e a ausência de dor, levando-se em consideração a alteração préexistente e o procedimento cirúrgico realizado.

\section{DISCUSSÃO}

A partir dos resultados encontrados nesta pesquisa, foram levantadas algumas considerações a respeito da hipertrofia do músculo masseter e de seu tratamento.

Quanto ao gênero, dos 4 pacientes estudados, 3 eram do gênero masculino e 1 do gênero feminino. Este achado mostra que houve uma prevalência do gênero masculino em relação ao feminino, o que discorda da literatura estudada, na qual não se observou distinção entre os gêneros $1,5,7,17,19$.

Em relação à idade, todos os pacientes dessa pesquisa se encontraram entre a faixa etária dos 19 e 24 anos. Este resultado mostra uma maior incidência da patologia na $2^{a}$ e $3^{a}$ décadas de vida, o que também concorda com muitos autores $^{1,3,5,7,8,8,17,19}$.
No que se refere ao procedimento realizado, todos os pacientes foram tratados cirurgicamente pelo acesso intrabucal. De acordo com este resultado foi possivel observar ausência de cicatriz e de comprometimento do nervo facial e o acesso direto ao feixe profundo do músculo. Cabe ressaltar que a opção da técnica cirúrgica utilizada dependerá da formação e da experiência de cada cirurgião.

Segundo Smyth ${ }^{17}$; Hakam, Beheiry e Helmy ${ }^{3}$; Souza, Freitas, Merida ${ }^{9}$; Mandel e Tharakan ${ }^{8}$, o tratamento realizado para hipertrofia do músculo masseter é cirúrgico por acesso intra ou extrabucal. Para Garcez, Santos ${ }^{1}$; Lasco ${ }^{4}$; Soares et al. ${ }^{18}$; Mandel e Kaynar ${ }^{7}$ o acesso extrabucal é preconizado, enquanto para Nishida e lizuka ${ }^{13}$ é o intrabucal.

As principais queixas referidas pelos pacientes no pós-operatório estiveram relacionadas à limitação da abertura de boca, à dor, à rigidez muscular e aos ruídos articulares. De acordo com Hakan, 
Beheiry, Helmy ${ }^{3}$ e Lasco ${ }^{4}$ estes são alguns dos sintomas mais encontrados no pós-operatório. Estes autores citaram ainda a presença do edema, porém este não foi observado como queixa nos pacientes desta pesquisa.

Como verificado na revisão da literatura, os autores priorizaram a descrição dos sintomas précirúrgicos, sendo a sintomatologia pós-cirúrgica referida apenas pelos dois autores citados anteriormente ${ }^{3,4}$.

No que se refere à etiologia, todos os pacientes apresentaram os hábitos deletérios de apertamento dentário e bruxismo como possiveis causas da patologia. Este resultado mostrou-se concordante com os de outros autores ${ }^{1,2,4,7,8,9,10,10,11,12,15,16,17,18,19,20}$ que descrevem os mesmos sintomas.

Durante a avaliação, a limitação da abertura de boca variou de 15 a $24 \mathrm{~mm}$, sendo que, dos 4 pacientes avaliados, 2 apresentaram desvio para um dos lados durante este movimento. Por não se encontrar trabalhos realizados sobre a reabilitação dos pacientes tratados cirurgicamente, não foram obtidos dados concordantes ou discordantes com estes resultados.

O período de terapia variou de 2 a 3 meses, com número mínimo de 6 sessões de terapias fonoaudiológicas e máximo de 10 sessões, sendo enfocados orientações, uso de compressas, alongamento da musculatura facial e cervical, exercícios de manipulação da musculatura intra e extrabucais, de alavanca de abertura forçada de boca e de órgãos fonoarticulatórios. A alta foi concedida após uma abertura de boca satisfatória e a eliminação das queixas dolorosas e de rigidez, sendo obtidos dados de 37 a 40mm de abertura de boca final e eliminação da sintomatologia referida anteriormente.

Hakan, Beheiry e Helmy ${ }^{3}$ descreveram a necessidade de exercícios passivos e ativos de abertura de boca concordando com o resultado descrito. Já Lasco ${ }^{4}$ não aponta a necessidade de acompanhamento terapêutico para o restabelecimento da plenitude dos movimentos mandibulares no pósoperatório.

\section{CONCLUSÃO}

Frente aos resultados obtidos, pode-se concluir que a terapia fonoaudiológica apresentou-se como uma possibilidade de complementação ao tratamento cirúrgico realizado, na adequação da amplitude dos movimentos mandibulares, assim como na eliminação dos sintomas presentes no pós-cirúrgico (dor, rigidez, tensão, ruído articular) e na conscientização dos hábitos deletérios, que são apontados pela literatura como possíveis fatores desencadeantes.

A intervenção fonoaudiológica é mais um recurso terapêutico quando a situação exige a recuperação do equilíbrio e a manutenção da saúde do sistema estomatognático, que além de participar de funções vitais, relaciona-se à estética. 


\title{
The miofunctional oral intervention in the surgery treatment for the masseter muscle hipertrophy
}

\begin{abstract}
Aim: the aim of this study was to describe miofunctional oral intervention in patients with Masseter muscle hipertrophy treated by surgery. Methods: the sample consisted of 4 patients, male and female, with ages between 16 and 24 years, with Masseter muscle hipertrophy treated by surgery. Results: the main complains on the postoperative were related to trismus, muscle rigidity, and clicking in the temporomandibular joint. The myofunctional oral therapy was based on orientation in the abnormal habits (bruxism, clenching) elimination, hyperthermia induced in the masseter muscle, oral muscles massage, facial and cervical muscles stretching, miofunctional exercises and passive and active jaw opening exercises. The results demonstrated adequate opening movement and elimination of habits and pain on the postoperative. Conclusion: miofunctional oral therapy is a possible complement to the surgery treatment
\end{abstract}

Key words: Masseter muscle hipertorphy. Myofunctional therapy. Speech. Language and hearing sciences.

\section{REFERÊNCIAS}

1. GARCEZ, F. J. A.; SANTOS, J. S. Hipertrofia do músculo masseter: revisão da literatura com apresentação de técnica cirúrgica optativa. ABO Nac, Rio de Janeiro, v. 5, p. 34-401, 1990.

2. GREENWOOD, L. F. Disfunção dos músculos da mastigação. In: ZARB, G. A.; CARLSSON, G. E.; SESSLE, B. J.; NORMAN D. M. Disfunção da articulação temporomandibular e dos músculos da mastigação. 2. ed. São Paulo: Ed. Santos, 2000. p. 256-269.

3. HAKAN, M. M.: BEHEIRY, M. G.; HELMY, E. S. Surgical management of benign masseteric hypertrophy (B.M.H.). Egypt Dent J, Cairo, v. 41, p.1435-1440, 1995.

4. LASCO, G. E. Cirurgia oral: 20 anos de cirurgia maxilo-facial. In: BOTTINO, A.; FELLER, C. Atualização clínica em Odontologia. 6. ed. São Paulo: Artes Médicas, 1984. p. 219-230.

5. VON LINDERN, J. J.; NIEDERHAGEN, B.; APPEL, T.; BERGÉ, S.; REICH, R. H. Type A botulinum toxin for the treatment of hypertrophy of the masseter and temporal muscle: an alternative treatment. Plast Reconstr Surg, Baltimore, v. 107, p. 327-332, 2001.

6. LUZ, J. G. C Alterações temporomandibulares e sintomatologia. In: BIANCHINI, E. M. G. Articulação temporomandibular: implicações, limitações e possibilidades fonoaudiológicas. 1. ed. Carapicuíba: Pró-Fono, 2000. p. 105-130

7. MANDEL, L.; KAYNAR, A. Masseteric hypertrophy. NY State Dent J, New York, v. 60, no. 7, p. 44-47, Aug./Sep. 1994

8. MANDEL, L.; THARAKAN, M. Treatment of unilateral masseteric hypertrophy with botulinum toxin: case report. J Oral Maxillofac Surg, Philadelphia, v. 57, no. 8, p. 1017-1019, Aug. 1999.

9. SOUZA, L. C. M.; FREITAS, R. R.; MERIDA, J. T. Assimetria de face. In: SOUZA, L. C. M. et al. Cirurgia Ortognática e Ortodontia. São Paulo: Ed. Santos, 1998. p. 146-155.

10. MORSE, M. H.; BROWN, E. F. Ultrasonic diagnosis of masseteric hypertrophy. Dentomaxillofac Radiol, Tokyo, v. 19, no. 1 , p.18-20, Feb. 1990

11. MOORE, A. P.; WOOD, G. D. The medical management of masseteric hypertrophy with botulinum toxin type $\mathrm{A}$. Br J Oral Maxillofac Surg, Edinburgh, v. 32, no. 1, p. 26-28, Feb. 1994.
12. NEWTON, J. P.; COWPE, J. G.; MCCLURE, I. J. Masseteric hypertrophy: preliminary report. Br J Oral Maxillofac Surg Edinburgh, v. 37, no. 5, p. 405-408, Oct. 1999.

13. NISHIDA, M.; IIZUKA, T. Intrabucal removal of the enlarged mandibular angle associated with masseteric hypertrophy. J Oral Maxillofac Surg, Philadelphia, v. 53, p. 1476-1479, 1995.

14. SANO, K. et al. Application of magnetic ressonance imaging and ultrasonography to preoperative evaluation of masseteric hypertrophy. J Craniofac Surg, New York, v. 19, p. 223-226, 1991.

15. SATOH, K. et al. Analyses of muscular activity, energy metabolism and muscle fiber type composition in a patient with bilateral masseteric hypertrophy. J Cranio-mandibular Pract, Chattanooga, v. 19, no. 4, p. 294-301, Oct. 2001.

16. SKOURA, C. et al. Masseteric hypertophy associated with administration of anabolic steroids and unilateral mastication: a case report. Oral Surg Oral Med Oral Pathol Oral Radiol Endod, St. Louis, v. 92, p. 515-8, 2001

17. SMYTH, A. G. Botulinum toxin treatment of bilateral masseteric hypertrophy. Br J Oral Maxillofac Surg, Edinburgh, v. 32, no. 1 p. 29-33, Feb. 1994

18. SOARES, M. M. et al. Hipertrofia Benigna do músculo masseter. Rev ABO Nac, Rio de Janeiro, v. 2, p. 200-202, 1994.

19. TO, E. W. H. et al. A prospective study of the effect of botulinum toxin A on masseteric muscle hypertrophy with ultrasonographic and electromyographic measurement. Br J Plast Surg, Edinburgh, v. 54, no. 3, p. 97-200, Apr. 2001.

20. TRUJILLO, R.; FONTÃO, F. N. G. K.; SOUZA, S. M. G. Unilateral masseter muscle hypertrophy: a case report. Quintessence Int Berlin, v. 33 , no. 10, p. 776-9, Dec. 2002.
Endereço para correspondência

aura Davison Mangilli

Rua Madalena, 523/ apto 41. Vila Madalena

CEP: 05.434-090 - São Paulo/SP

E-mail: davisonmangilli@yahoo.com.br 